\title{
Las migraciones de retorno de la población ecuatoriana y boliviana: motivaciones, estrategias y discursos
}

\author{
Cristina VEGA SOLÍS \\ Profesora Investigadora, FLACSO-Ecuador \\ cvegas@flacso.edu.ec \\ Raquel MARTÍNEZ-BUJÁN \\ Universidade da Coruña \\ rmbujan@udc.es
}

Recibido: Junio 2015

Aceptado: Junio 2016

\section{RESUMEN}

Este artículo analiza las motivaciones de las migraciones de retorno de los colectivos ecuatorianos y bolivianos que habiendo residido en España han regresado a sus comunidades de origen durante la etapa de crisis económica iniciada en el año 2008. A partir del análisis de 22 entrevistas en profundidad realizadas en Ecuador y 38 en Bolivia a mujeres, hombres y jóvenes pertenecientes a familias migrantes, se demuestra que este proceso de toma de decisión se incrusta en una dinámica relacional de género. En concreto, se indaga en los elementos afectivos y económicos que influyeron en la decisión de retornar, así como en las estrategias desplegadas para proyectar su reacomodo en origen. Hombres y mujeres ocupan posiciones diferenciales en el entorno familiar, laboral y social, estando sus expectativas construidas de manera generizada. Si bien la migración ha supuesto para las mujeres un mayor poder económico dentro del grupo familiar, su reinserción en el retorno redefine su papel como gestoras principales de la unidad doméstica y de las dinámicas que permiten su reproducción social. Por su parte, los hombres aspiran a reactualizar su papel como proveedores a pesar de su frágil situación laboral al regreso. La movilidad social para ellas se transfiere generacionalmente a través de una fuerte inversión educativa proyectada sobre sus hijas e hijos, mientras que para ellos, ésta se articula en la creación de negocios familiares y su capacidad demostrativa.

Palabras clave: Migración de retorno, género, cuidados, emprendimiento, motivaciones. 


\title{
Return Migration in the Ecuadorian and Bolivian population: motivations, strategies and discourses
}

\begin{abstract}
This article analyses the motivations for return migration among the Ecuadorians and Bolivians who, after living in Spain, returned to their countries of origin during the economic crisis that started in 2008. From the analysis of 22 interviews in-depth which took place in Ecuador and 38 in Bolivia to women, men and young people from migrant families, this decision-making process is shown to be embedded into a gendered dynamics of relationships. Particular detail is given to affective and economic elements that had an influence on the decision to return, as well as to the strategies deployed to project their readjustment back in origin. Males and females occupy differential positions within the family, work and social circle, their expectations being built in a gendered manner. Despite the fact migration has brought women greater economic power within the family group, their reintegration upon return redefines their role as main managers in the household and the dynamics that allow their social reproduction. Men, for their part, aspire to refresh their role as providers in spite of their frail labour position upon return. Social mobility for females is passed on through generations by a strong investment on education for their daughters and sons, while for males this mobility revolves around setting up family businesses and around their demonstrative abilities.
\end{abstract}

Keywords: Return migration, gender, care, enterpreneurship, motivations.

\section{INTRODUCCIÓN}

Los estudios relacionados con las migraciones de retorno han proliferado especialmente en las etapas de crisis económicas y muchas de las contribuciones académicas en este ámbito han intentado estudiar cuáles eran sus repercusiones sobre el desarrollo económico en las comunidades de origen. De esta manera, la literatura científica ha sido proclive a vincular el concepto de retorno al éxito económico y social adquirido con la migración, a los efectos que se pueden generar las etapas de recesión y a la capacidad que tendrá este colectivo de potenciar, a través de sus recursos, el desarrollo de sus localidades de partida. Estos planteamientos tradicionalmente vinculados a las teorías neoclásicas (revisiones seminales pueden consultarse en De Haas 2012, Cassarino 2004) parece que han comenzado a ser superados, al menos teóricamente. La comunidad académica hace décadas que está renovando sus marcos analíticos, por ejemplo, a través de las teorías transnacionales (Portes et al. 1999) o las de redes sociales (Massey 1990) y dejando en desuso las anteriores consideraciones. No obstante, durante la actual etapa de gran recesión (iniciada en el año 2008), los estudios sobre retorno y la influencia de la economía en este proceso han experimentado un resurgimiento (Plewa 2009). Estas crisis sistémicas y globales se siguen presentando como una oportunidad para estudiar nuevas contribuciones de la economía a las movilidades de las personas. Sin embargo, la mayoría de estas orientaciones al centrar su atención en las repercusiones de las contracciones económicas sobre los flujos y el mercado laboral, suelen omitir la influencia diferencial 
que ambos procesos experimentan entre hombres y mujeres e ignoran la relevancia de otras dimensiones que también operan en la decisión, tales como la influencia del ciclo de vida familiar o las tensiones en la organización de los cuidados transnacionales.

Este documento se ha construido precisamente para demostrar que, si bien la coyuntura económica y el mercado laboral pueden influir en la toma de la decisión de regresar, dichos factores muestran repercusiones desiguales con respecto al género y el país de origen. Mediante un análisis de las experiencias de las migraciones de retorno de la población boliviana y ecuatoriana asentada en España se demuestra que estos desplazamientos de vuelta están muy condicionados por cambios en los ciclos familiares, así como por conflictos y tensiones en la organización de los cuidados en los hogares que complejizan la centralidad de los aspectos económicos. La toma de la decisión de retornar no está condicionada únicamente por alteraciones en los mercados laborales, sino que la trayectoria migratoria también está mediada por las dinámicas familiares. La comparación entre las experiencias de retorno de los migrantes bolivianos y ecuatorianos visualiza la importancia del grado de asentamiento en destino y de los cambios estructurales en los países de origen tanto para proceder a la toma de la decisión como en la capacidad de movilización de los recursos necesarios para volver a casa.

El artículo se divide en cuatro apartados. En el primero de ellos, se presenta el marco teórico, y se justifica la necesidad de estudiar las migraciones de retorno desde una perspectiva de género que contemple las dinámicas personales y familiares en el retorno y el reacomodo en origen. En estos procesos, el lugar diferencial de hombres, mujeres y jóvenes en relación a los trabajos, a las responsabilidades familiares, a la educación o al reencuentro con las gentes y el país de procedencia juega un papel importante. El segundo apartado contextualiza las migraciones bolivianas y ecuatorianas hacia España para comprender su diferente posición con respecto al conjunto de las migraciones latinoamericanas. En este sentido, es preciso analizar la especificidad de sus desplazamientos de retorno y evaluar el impacto de la crisis económica en los mismos. En el tercer apartado se explica la metodología utilizada. Por último, en la cuarta sección, se exponen los hallazgos y se exploran los discursos sobre las razones y estrategias que los propios migrantes aducen para regresar y reinstalarse en el país que dejaron años atrás. Es aquí donde se muestra cómo las narrativas del retorno revelan interpretaciones patriarcales acerca de la participación de sus protagonistas en las responsabilidades reproductivas y productivas.

\section{MIGRACIÓN Y RETORNO EN UNA PERSPECTIVA DE GÉNERO Y GENERACIÓN}

El aporte de la perspectiva de género al análisis de la migración internacional se ha producido a distintos niveles a lo largo de las últimas tres décadas (Herrera 2013), aunque cabe mencionar que dentro de este campo de estudio las exploraciones sobre cuáles son las razones que coartan o animan las movilidades han sido menos abordadas respecto a otras dimensiones de la migración. En un primer nivel, que comienza a mediados de los años ochenta, los estudios se centraron en visibilizar las experiencias 
migratorias femeninas y el papel de las mujeres como sujetos activos en los desplazamientos. De esta manera, se puso de relieve la presencia diferencial de éstas en los procesos migratorios y el carácter autónomo de sus movilidades. En un texto ya célebre de los años ochenta, Mirjana Morokvasic (1984) llamaba la atención sobre los crecientes desplazamientos de mujeres empobrecidas para insertarse como mano de obra flexible, vulnerable y barata en los nuevos entornos capitalistas en varias regiones del planeta. Estos planteamientos se consolidaron en la discusión académica y desde finales de la década de 1970 no han cejado de producirse exploraciones que ponen de manifiesto la denominada "feminización de las migraciones" en el contexto de la globalización (Gamburd 2000).

En un segundo nivel, los aportes se relacionan con el modo en el que la sociedad produce el género y el poder generizado (casi siempre apelando a su carácter natural) como un modo de fundamentar la desigualdad (Izquierdo 2013). Dicha regulación, se apoye o no en elementos discriminatorios instituidos, se manifiesta de forma conexa en el mercado laboral, la familia, la educación, la cultura y, en general, en todos los ámbitos de la vida social. Las dinámicas migratorias no son ajenas a esta producción de género como desigualdad, por lo que estos estudios utilizaron las migraciones para analizar la transformación de la feminidad y la masculinidad. En este sentido, éstas pueden ser leídas como un proceso de dirimir, no siempre de forma armónica, democrática, ni siquiera consciente, qué y quiénes pesan más a la hora de hacer las maletas y qué lugar jugará cada cual en el nuevo esquema tras el desplazamiento (Mahler y Pessar 2001). También en la migración se ponen en juego las relaciones de poder y las atribuciones que se hacen a unas y a otros, especialmente en lo tocante a las responsabilidades familiares, el trabajo asalariado, las relaciones y modos de participación en las sociedades de llegada o las formas de identificación como nacionales de cierto país o adscritas a cierto grupo étnico. El ingente volumen de investigaciones acerca de la presencia de mujeres migrantes en los nichos del empleo de hogar y cuidados revela este tipo de dinámicas, en las que se refuerza una feminidad singular disponible, afectuosa, servil-, con rasgos étnicos y nacionales, encarnada por mujeres dispuestas a darlo todo a cambio de bien poco para responder a los nuevos arreglos familiares y laborales entre la población en los países de destino (Anderson 2000).

Junto a la (re)producción de identidades desiguales de género en la migración, se dan procesos de empoderamiento a diferentes escalas, hecho que impide pensar que exista un vínculo lineal o polarizado en este sentido (Anthias 2000, Mahler y Pessar 2001). En un reciente trabajo sobre la migración desde comunidades rurales en México, Patricia Arias (2013) explica cómo para las mujeres casadas reunirse con sus esposos en los Estados Unidos, en un contexto de retorno incierto, es una estrategia para escapar al férreo control que ejercen los suegros en un esquema de residencia patrilocal que les impone el deber de ayudar y cuidar de su familia política. Este caso muestra cómo el elemento generacional se articula con el género y cómo el control de los mayores sobre los jóvenes, en particular sobre las jóvenes, puede ser un elemento clave para moverse. Migrar para "estar junto a sus esposo" se convierte así en un argumento legítimo para huir de las asignaciones intergeneracionales de género. 
La agencia femenina se juega en una compleja dinámica, que como muestran los estudios sobre la familia, no se dirime únicamente en destino u origen, sino en circuitos transnacionales (Pedone 2006, Herrera 2012, 2013). Este tipo de análisis pueden ubicarse en un tercer nivel, que algunas autoras como Herrera (2013) denominan "interseccional”, en el sentido de que el género se articula con otros ejes de la desigualdad social como la clase, la etnia o la edad. Estas investigaciones se centran en averiguar cómo interseccionan estas tres categorías a partir de las cuáles se marcan claras desventajas sociales y de qué manera interactúan en conjunto para explicar la experiencia de discriminación de las mujeres (Bastia 2014). Desde este ámbito interseccional estudiado con una perspectiva transnacionalista es donde han encajado con más éxito los análisis de las migraciones de retorno y la diferente forma que adquiere este proceso según el género. De hecho, Luis Guarnizo (1997), uno de los autores principales de la corriente transnacionalista, reconoce en su texto "Going home: class, gender and household transformations among Dominican return migrants”, que uno de los avances teóricos más relevantes en el análisis del retorno es precisamente que no puede definirse como un concepto estático ni tampoco unidireccional. Es decir, que el retorno forma parte del proceso de la migración pero no tiene que suponer su fin, sino que puede ser un paso en la trayectoria de movilidad que conecta destino y origen. Un análisis transnacional del retorno supone cuestionar su definición tal y como la habían considerado las teorías clásicas y su vinculación exclusiva con aspectos económicos o el éxito alcanzado en la sociedad de destino. Las mujeres, hombres, adultos y jóvenes, inmersos en procesos migratorios singulares son capaces de actuar de acuerdo a una rica articulación de motivaciones; éstas no siempre son producto de deliberaciones racionales o acordadas en las parejas o las familias. Así, huir de una relación de pareja violenta o sencillamente asfixiante o evitar un destino marginal para la siguiente generación se conforman como motivos tan buenos para migrar como lograr un nivel económico aceptable. En ocasiones, el economicismo de los análisis no ha dejado ver estos otros aspectos de índole afectiva, sexual, cultural o política, que indudablemente no pueden ser desligados de las dimensiones económicas. Situar el peso en los factores económicos revelaba, en realidad, un sesgo en la mirada: el que se deriva de primar la asociación entre migración, varones y varones adultos como sustentadores vinculados al mercado (Hernández et al. 2012). Contemplar la migración desde las mujeres, desde las poblaciones con una frágil inserción laboral, desde las y los jóvenes y, desde luego, desde las niñas y los niños, arroja otros resultados. La creciente presencia de investigaciones sobre las hijas e hijos de la migración y la reproducción de la desigualdad en la experiencia de los jóvenes, entrecruzada con el género, ha sido una enorme aporte en campos como las trayectorias formativas, educativa y laboral (García 2003, Pedreño 2013), los procesos de autoidentificación nacional y étnica en la migración (Moscoso 2013, Celleri 2011) o las discriminaciones racistas.

Estas recientes exploraciones están contribuyendo también a repensar las migraciones de retorno. Estudios recientes ponen de relieve que las motivaciones para volver y la toma de la decisión no siempre están ancladas al trabajo y al dinero, y que mujeres y hombres sopesan y enfatizan de forma dinámica distintos aspectos de su existencia en la migración (Rivera 2011). Peter Hansen (2008), en su estudio del 
retorno de hombres y mujeres somalíes, o Nicole Constable (1999), en el de mujeres filipinas, advierten que las mujeres se muestran ambivalentes ante la vuelta a casa temiendo que su regreso implique una pérdida de autonomía, hecho que también se señala en otros trabajos. Las mujeres, en general, tienden a poner el acento en sus obligaciones de cuidado y en la proyección educativa y profesional de hijas e hijos. Esto último es corroborado en las recientes investigaciones de K. F. Olwig (2012) sobre mujeres caribeñas y de Ionela Vlase (2013) centrada en el retorno de mujeres rumanas desde Italia. La maternidad, frente a los logros económicos, más aducidos por los varones, se presenta en estos casos como una fuerte motivación para volver.

Los análisis sobre el retorno a los países andinos desde los actuales contextos europeos de crisis arrojan reflexiones interesantes en clave de género. Por una parte, se relativiza el peso de la crisis en la motivación para regresar, especialmente en las etapas iniciales de la misma (Bastia 2011a). Y no es que ésta no tenga importancia en lo que se refiere a la degradación del empleo y las prestaciones, sin embargo, su influencia no se refiere únicamente a estos aspectos. Por ejemplo, para el caso de Bolivia, Leonardo de la Torre (2011), revisando los elementos que inhiben y potencian la vuelta a casa, señala que la reagrupación familiar es un elemento que incide en la decisión. Aunque no se detiene en explicar la variabilidad de su relevancia según sexos, reconoce la existencia de diferencias significativas. La crisis es una realidad multidimensional cuyas afectaciones deben ser leídas desde distintos ángulos. A partir de una encuesta realizada en Cataluña, Sonia Parella (2012) también advierte razones diferenciales según el género para el regreso a Bolivia desde España. De acuerdo con Carmen Ledo (2014), el 78,6\% de las mujeres de esta nacionalidad aducen causas familiares, frente, por ejemplo, el 4,9\% que se refieren a la falta de empleo.

En el caso ecuatoriano, en el estudio pionero de Hernández et al. (2012) sobre migración y retorno en los barrios populares del noroccidente de Quito, las autoras ponen de relieve las múltiples estrategias que se ponen en marcha para mantenerse en destino. En los distintos retornos identificados -temporal, de prueba, planificados (en principio, definitivos), impulsados desde las instituciones o por cuenta propia- existe una preeminencia del cuidado como motivación para regresar en el caso de las mujeres (32\% frente al 18\% de varones). El ciclo de vida de la prole condiciona el retorno incluso en aquellas ocasiones en que el proyecto se estabiliza en destino. Cuestiones culturales y valores morales acerca del futuro de los hijos juegan un enorme peso. Los hombres, por su parte, destacan las dificultades laborales como principal causa para regresar (54\%). Si la familia está separada y el dinero ya no alcanza más que para mantenerse, el regreso se torna la salida más razonable tras largos años de separación.

Otra investigación sobre retorno a Llano Grande, una comuna urbana del norte de Quito, realizada por Gioconda Herrera y Lucía Pérez, muestra que alrededor del 50\% de los retornados enfatiza el deseo de estar junto a la familia, frente al $29 \%$ que habla de falta de empleo en destino como principal causa del retorno, otro 17\% afirma haber regresado al cumplir sus objetivos económicos (Herrera y Vega 2013). Los fuertes vínculos transnacionales desarrollados por los llanograndenses hacen pensar en migraciones de ida y vuelta cíclicas y temporales, que más que de retorno hablan de existencias transnacionales. Las autoras enfatizan el repliegue en el hogar de las 
mujeres tras su inserción laboral en España. Entre los aspectos claves en el regreso, este estudio llama la atención sobre el papel que juega el capital cultural y económico antes de la partida en los procesos de reinserción. La segmentación del mercado de trabajo en destino hace que el peso de lo acumulado en la migración se refiera más a activos económicos y financieros, especialmente dirigidos a Ecuador, que a la educación.

Tal y como explicaremos, la presente investigación pone igualmente de manifiesto las diferentes formas en las que mujeres, hombres y jóvenes de ambos sexos experimentan el retorno. Veremos cómo el ciclo de vida familiar, la apuesta por el emprendimiento y la composición de las familias son elementos claves a la hora de dar cuenta de las razones, estrategias y expectativas para salir y reinstalarse en destino. Desde esta perspectiva, la decisión de retornar se conecta, más allá de los mercados laborales, con la experiencia vital de los integrantes de la familia.

\section{CONTEXTOS DE MIGRACIÓN A ESPAÑA DESDE ECUADOR Y BOLIVIA Y ESPECIFICIDADES EN SU RETORNO}

Las migraciones de las poblaciones ecuatoriana y boliviana hacia España son un fenómeno relativamente reciente. El asentamiento se ha producido especialmente durante la primera década del 2000 cuando España se consolida como el principal país de recepción de los flujos migratorios latinoamericanos en el entorno de la OCDE. La expansión económica española junto con unas leyes de migración tolerantes fueron los detonantes principales para la instalación de ambos colectivos (Bertoli y FernándezHuertas 2013). Es en esa etapa cuando los datos del Padrón Municipal de Habitantes evidencian el vigor de la corriente ecuatoriana. Antes de 1999 tan sólo unos 76.000 ecuatorianos vivían en España, mientras que a comienzos de 2005, los residentes ya alcanzan los 497.799. La Estadística de Variaciones Residenciales, que contabiliza los flujos migratorios, estima la entrada de 91.000 ecuatorianos en el año 2000, 82.000 en el 2001 y 88.000 en el año 2002. Es decir, la fase explosiva de sus llegadas se produce entre el año 2000 y el 2003. El ritmo se desacelera bruscamente desde el 2004 con la imposición de visado a los nacionales de Ecuador y a partir del 2010 su número mengua hasta situarse en el año 2014 en torno a los 219.000 residentes. Aún así, Ecuador representa el tercer país de la inmigración en España (sólo por detrás de Marruecos y Rumanía) y las cifras de ecuatorianos con nacionalidad española indican el alto grado de asentamiento en el país. Los datos más recientes señalan la residencia de más de 453.000 españoles nacidos en Ecuador.

El asentamiento de la población boliviana es posterior en el tiempo. De hecho, la fortaleza de su crecimiento aparece justamente tras la ralentización del flujo migratorio ecuatoriano $\mathrm{y}$, al igual que éste, se caracteriza por su intensidad en un corto espacio de tiempo. Su instalación tiene lugar durante la etapa 2005-2007. En el año 2001 se contabilizan tan solo a unas 6.600 personas procedentes de Bolivia, pero en el año 2005 se registran ya 97.497. La cifra sigue aumentando en unas 40.000 personas anuales hasta el año 2008, cuando se estima la residencia de 242.496 habitantes de nacionalidad boliviana. Según los últimos datos consultados (año 2014), en la actualidad viven en 
España alrededor de 150.000 bolivianos $^{1}$. La exigencia de visado aprobada en el año 2007 también ha templado el ritmo de sus llegadas desde esa fecha. Precisamente, dicha medida coincide con el comienzo de la crisis económica española, que se ha traducido en una mayor rigidez para conseguir la estabilidad de la residencia, así como en un control más férreo de las entradas, por lo que al mismo tiempo que desciende la población boliviana que entra, también aumentan sus salidas hasta el punto de que Bolivia se convierte en la primera nacionalidad con saldo migratorio negativo.

En la Figura 1, que representa la evolución del saldo migratorio de los principales países de inmigración latinoamericana en España, puede observarse esta tendencia. Las entradas descienden de forma abrupta a partir de la Gran Recesión y los flujos de salida se acentúan pero con intensidades desiguales entre las nacionalidades. De esta manera, el saldo migratorio (medido como la diferencia entre las entradas y las salidas) de los países de mayor tradición de asentamiento (como República Dominicana) se mantiene durante todo el período de crisis aunque con respecto a Bolivia, la pérdida de sus residentes comienza desde los inicios del decrecimiento en el año 2008 y se hace más fuerte en los siguientes dos años hasta asentarse en una pérdida de población de entorno 10.000 personas anuales desde el 2011. Para la población ecuatoriana la resistencia al retorno aguanta hasta el año 2010. A partir de ahí el volumen de salidas comienza a ser tan significativo que el saldo migratorio se convierte en negativo. Y la tendencia continúa hasta la actualidad. Estos ritmos señalan unas tasas de retorno (el número de personas que se marchan por cada cien empadronados) muy vigorosas para la población boliviana, sobre todo, en los primeros años del decrecimiento y menos potentes para los ecuatorianos, al menos hasta que la persistencia de la crisis empieza a percibirse y hace mella hasta en los colectivos más asentados. En el año 2013 abandonan el país el 10,0\% de los residentes bolivianos mientras que la cifra de los ecuatorianos alcanza el $7,7 \%^{2}$.

El nivel de arraigo parece ser la explicación más plausible de esta incidencia desigual del retorno entre ambas poblaciones (Izquierdo y Martínez-Buján 2014). Con independencia de los proyectos migratorios, la llegada más reciente de los flujos migratorios bolivianos ha influido en su menor estabilidad jurídica, en su dificultad para reagrupar a la familia y en su más precaria posición en el mercado laboral, procesos que influyen en la decisión de regresar (Jones y De La Torre 2011). Es decir, el tiempo de permanencia en España de ambos colectivos y lo que de ello se deriva ha determinado condiciones diferenciales en el acceso a los canales básicos de asentamiento tales como la nacionalidad, la residencia permanente o contratos laborales estables y con mejores condiciones. Aunque en el año 2014, los datos indican que el porcentaje de migrantes bolivianos con Permiso de Residencia de Larga Duración se sitúa en el 51,1\%, en el período inicial de la crisis (año 2008) esta cifra era del 6,8\%. Los residentes ecuatorianos con residencia permanente alcanza ya el 86,8\%, mientras que al comienzo de la recesión éstos se situaban en el 30,3\%. De esta manera,

\footnotetext{
${ }^{1}$ Datos recabados del Instituto Nacional de Estadística, Padrón Municipal de Habitantes.

${ }^{2}$ Los datos sobre entradas y salidas de población extranjera proceden del Instituto Nacional de Estadística, de la Estadística de Variaciones Residenciales.
} 
el 53,4\% de los ecuatorianos ya han obtenido la nacionalidad, mientras que el dato desciende al 20,3\% en el caso del colectivo boliviano (consultar datos en tabla 1). En cuanto a la reagrupación familiar, la Encuesta Nacional de Inmigración señala que la migración boliviana contiene la proporción más alta de familias separadas: el 36\% de los varones y el $21 \%$ de las mujeres casadas que viven en España no conviven con su pareja. En el segmento de aquellos que tienen hijos (un 32\% de los emigrados), más de la mitad los dejaron en su país (Baby-Collin y Cortés 2014). Esta situación contrasta con la de la población ecuatoriana, donde el $43,9 \%$ de sus residentes tiene una edad de entre 16 y 24 años (Colectivo IOÉ y Fernández 2010).

Figura 1. Evolución saldo migratorio (entradas-salidas) de población en España según nacionalidad, 2002-2013.

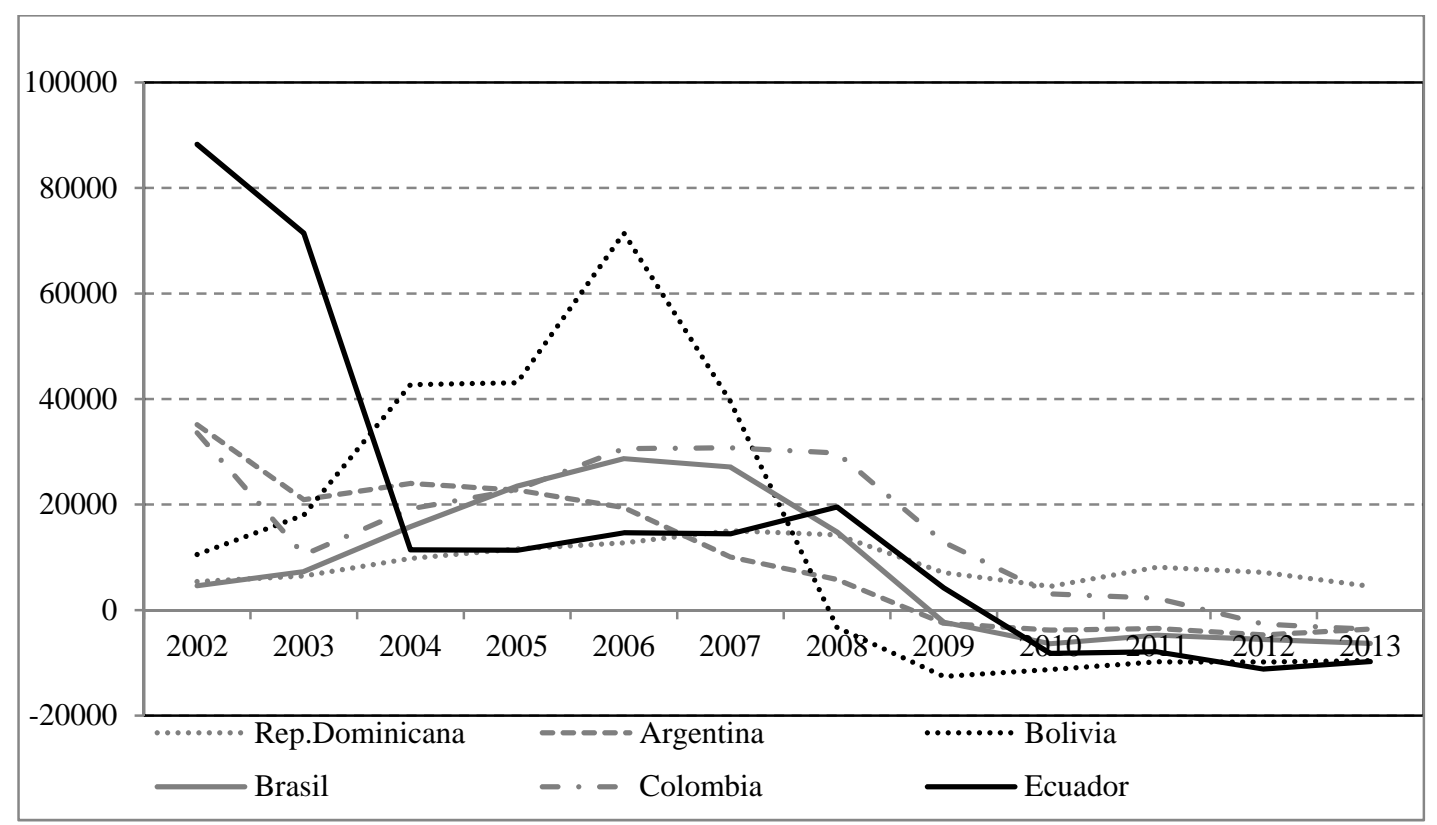

Fuente: Elaboración propia, INE, Estadística de Variaciones Residenciales.

Por otra parte, algunas investigaciones (Colectivo IOÉ 2012) han puesto de manifiesto que la población boliviana masculina ha sido el colectivo de América Latina residente en España más afectado por el decrecimiento debido a su fuerte vinculación al sector de la construcción. Siguiendo los datos de 2014 de la Encuesta de Población Activa española, la tasa de paro de los hombres bolivianos alcanza la cifra del 38,3\%, cuando entre los ecuatorianos es del 31,3\%. Los datos muestran una mejor integración en el mercado de trabajo entre las mujeres de ambas nacionalidades. Esto se debe a la mayor resistencia de los empleos en el servicio doméstico y de cuidados a los que acceden las mujeres. Antes de la crisis, se ocupaban en esta actividad el $64,8 \%$ de las bolivianas, mientras que en el año 2014 su concentración en el sector alcanza al 75,6\%. Las mujeres ecuatorianas también desempeñaron esta ocupación, en mayor medida al 
comienzo de su asentamiento en el país, por lo que durante la etapa de recesión ya habían experimentado una clara movilidad laboral hacia otros sectores (Herrera 2012). Aun así, desde la crisis su presencia en el sector ha aumentado. Los últimos datos señalan que el $36,4 \%$ de las mismas trabajan como empleadas de hogar. Aunque en esta fase la tasa de desempleo femenino entre el colectivo boliviano ha experimentado un notable incremento alcanzando el 15,5\% en 2014, entre las mujeres de Ecuador la cifra aumenta considerablemente hasta el $24,6 \%$. Trabajar en el servicio doméstico parece que amortigua los efectos del desempleo. Los hombres, por el contrario, se incorporaron específicamente a la construcción, donde se produjo la mayor destrucción de empleos. Si en el año 2008 el 54,5\% de los hombres bolivianos y el 43,5\% de los ecuatorianos se ocupaban en esta actividad, en el año 2014 ya tan sólo se ubicaban el 11,5\% y el 12,5\% respectivamente (consultar datos en tabla 1).

Tabla 1. Indicadores de la evolución del asentamiento de los migrantes ecuatorianos y bolivianos en España.

\begin{tabular}{|c|c|c|c|}
\hline & Ecuador & Bolivia & Fuente de datos \\
\hline Período de llegada a España & $2000-2003$ & 2005-2007 & Estadística de Variaciones \\
\hline Tiempo medio de residencia en & 10 años & 4 años & Residenciales y Padrón \\
\hline Año de salidas más elevado & 2009 & 2013 & \\
\hline \% residentes Larga Duración & 86,7 & 51,1 & $\begin{array}{l}\text { Anuario Estadístico de } \\
\text { Inmigración (año 2013) }\end{array}$ \\
\hline $\begin{array}{l}\text { \% personas con nacionalidad } \\
\text { española }\end{array}$ & 53,4 & 20,3 & $\begin{array}{l}\text { Padrón Municipal de } \\
\text { Habitantes (año 2014) }\end{array}$ \\
\hline Tasa de paro hombres & 31,3 & 38,3 & \\
\hline Tasa de paro mujeres & 24,6 & 15,5 & Encuesta de PoDiacion \\
\hline \% mujeres en servicio doméstico & 36,4 & 75,6 & Activd \\
\hline \% hombres en construcción & 12,5 & 11,5 & \\
\hline Tasa de retorno hombres & 8,3 & 11,8 & $\begin{array}{l}\text { Estadística de } \\
\text { Variaciones }\end{array}$ \\
\hline Tasa de retorno mujeres & 7,0 & 8,7 & $\begin{array}{l}\text { Residenciales y Padrón } \\
\text { Municipal de Habitantes }\end{array}$ \\
\hline
\end{tabular}

Fuente: Elaboración propia.

El trabajo de campo muestra evidencias de esta precariedad laboral vivida entre los hombres y mujeres bolivianas y ecuatorianas y no puede obviarse que esta drástica pérdida de empleos ha provocado motivos, diferenciales según el género, para regresar a origen. Pero, la influencia del empleo en esta vuelta a casa y, en general, la economía es utilizada de distintas maneras en los discursos de hombres y mujeres. De hecho, las tasas de retorno según el género en cada una de las nacionalidades no presentan la pauta que cabría esperar, dados sus niveles de desempleo. De esta manera, la de las mujeres bolivianas que se sitúa en un $8,7 \%$ es superior a la de sus homólogas ecuatorianas (un $7,0 \%$ ) a pesar de su mejor inserción laboral, hecho que induce a 
pensar que existen otros elementos a parte de los económicos que impulsan la decisión de retornar o de quedarse.

\section{METODOLOGÍA}

El presente artículo está basado en dos investigaciones realizadas, entre 2008 y 2012 en el caso de Bolivia, y entre 2013 y 2014 en el de Ecuador ${ }^{3}$. Tal y como veremos, el impacto de la evolución de la crisis en las dinámicas de retorno es, en relación a los períodos cubiertos, un aspecto relevante a la hora de captar motivaciones y estrategias de parte de los actores, puesto que si bien en un primer momento el retorno no aparece como una opción preferente, especialmente en el caso de la población ecuatoriana, mucho más asentada, en una segunda fase y ante situaciones de desgaste, se torna en una opción más aceptable.

En ambas investigaciones se realizaron entrevistas semi-estructuradas a mujeres, hombres y jóvenes retornados o, en el caso de los ecuatorianos, en procesos de retorno, con el fin de captar las motivaciones y estrategias a la hora de desplazarse y reubicarse en origen. En Ecuador, las entrevistas se hicieron en Quito, mientras que en Bolivia se llevaron a cabo en dos ciudades: Cochabamba y La Paz, en ambos casos, de localizaciones relevantes en la migración hacia España. En Bolivia se hicieron un total de 38 entrevistas (13 hombres y 25 mujeres) a migrantes retornados y 10 entrevistas a técnicos de ONG's en dos períodos (el primero en el año 2010 y el segundo en el año 2012), mientras que en Ecuador se llevaron a cabo 22 entrevistas multisituadas correspondientes a 8 familias, algunas ya totalmente de vuelta y otras aún con miembros en España. En ambos casos, las personas entrevistadas responden al perfil en cuanto a tiempo de estancia, período de salida y recorrido migratorio- que caracteriza a ambos colectivos. En el caso de Ecuador, un rasgo distintivo de las personas entrevistadas se refiere a la percepción de la ayuda estatal -el fondo Cucayopara poner en marcha un emprendimiento. Siendo uno de los requisitos de este programa el contar con un capital semilla, entendemos que este grupo no es el más castigado por la crisis, ya que en general pudieron salir de España si no de forma necesariamente planificada, sí al menos con algunos ahorros. A pesar de las diferencias en la estrategia metodológica -en Bolivia, dos fases de trabajo de campo en origen, en Ecuador, un período menor pero con trabajo de campo transnacional-, consideramos que las entrevistas proporcionan insumos claves para un análisis de carácter

\footnotetext{
${ }^{3}$ La investigación sobre Ecuador fue parte del proyecto El retorno a Ecuador desde España: Estrategias económicas, sociofamiliares y afectivas para una integración transnacional, financiado por Flacso-Ecuador. Se realizaron dos estudios de caso, uno sobre los retornados beneficiarios de fondo Cucayo, coordinado por Cristina Vega, y otro, sobre el retorno en Llano Grande, coordinado por Gioconda Herrera; participaron además, Maria Isabel Moncayo, Grégory Dallemagne y Lucía Pérez. La desarrollada en relación a Bolivia estuvo a cargo de Raquel Martínez Buján y se corresponde a la investigación Proyectos y procesos de retorno de los inmigrantes extranjeros en España, financiada por el Ministerio de Ciencia e Innovación de España, y coordinada por Diego López de Lera.
} 
comparativo en la medida en que elaboran el retorno en la coyuntura de la crisis explorando las motivaciones y procesos que llevaron a esta decisión.

En Bolivia, se accedió a los entrevistados a través de los contactos facilitados por organizaciones no gubernamentales y asociaciones dedicadas a la gestión de las migraciones internacionales. También se utilizó la técnica la bola de nieve. En Ecuador, los contactos con los entrevistados parten de una base de datos facilitada por la SENAMI en la que se recogen aquellos emprendedores financiados por la administración. A partir de una primer contacto se pudo entrevistar con otros miembros de la familia, tanto en Ecuador como en España. En ambos casos, la extensión de las entrevistas varía entre una y tres horas y todas ellas fueron grabadas y transcritas. Se recogió información sobre los siguientes temas: migración y experiencia laboral en España, acomodos familiares, prácticas transnacionales, organización social de los cuidados, toma de decisión del retorno, oportunidades tras el retorno, y cambios autopercibidos en la identidad. El trabajo de campo se desarrolló en áreas urbanas y periurbanas y la mayoría de las personas entrevistadas únicamente tenía terminados sus estudios primarios en el caso de Bolivia, mientras que entre los ecuatorianos el nivel educativo se eleva. Entre las personas bolivianas, su posición económica antes de la migración es definida por ellos mismos como muy precaria y vulnerable, con salarios muy bajos y con la percepción de tener muy pocas oportunidades de mejorar en términos laborales. Los ecuatorianos, a diferencia de sus vecinos, tienden a describir su posición en una línea ascendente, llegando algunos de ellos a definirse como personas que "ganaron mucho dinero" y que hoy se ven perjudicados por la crisis.

\section{MOTIVACIONES Y ESTRATEGIAS EN EL RETORNO A BOLIVIA Y ECUADOR DESDE ESPAÑA}

\subsection{CRISIS, TRABAJO Y VIDA FAMILIAR COMO RAZONES PARA PARTIR}

La coyuntura económica y laboral no ha facilitado la permanencia en España a medio plazo. Los datos mencionados en torno a las tasas de desempleo y precarización laboral, además del recorte en los servicios, han afectado las trayectorias migratorias en ambos colectivos. No obstante, teniendo en cuenta los motivos que los propios entrevistados mencionan en la toma de decisión no cabe reducirlos a este aspecto de la vida económica (Vega 2014; Martínez-Buján 2015). Tanto en las razones aducidas para regresar como en las estrategias llevadas a cabo para organizar la vuelta a casa, la vulnerabilidad vivida en España o el encauzamiento de nuevos proyectos profesionales en origen se entretejen con dinámicas afectivas y familiares que dotan de una mayor complejidad a los procesos de retorno. A esto se suma que el peso que adquiere la articulación de estos elementos en las decisiones se construye de manera diferente según el género y la edad.

Mientras que entre los hombres adultos predomina la construcción del retorno asociado a "montar un negocio" y retomar oficios o actividades anteriores, algo que 
suelen asociar a las dificultades laborales vividas en España, para las mujeres, las necesidades de las familias en origen o la proyección de hijas e hijos aparecen como razones preferentes que modelan el curso del proceso. En este último caso existe una prevalencia de las consideraciones concernientes a la salud, los cuidados (tanto de los hijos/as como de los progenitores) y las oportunidades educativas o profesionales de la siguiente generación. Mujeres y hombres asocian el regreso con la vida familiar, sin embargo, si bien para los varones ésta se cifra en su papel de proveedores económicos, para las mujeres, que aluden de igual modo a la degradación de las condiciones laborales que experimentaron en los últimos años, la atención se centra en el porvenir de la familia en aspectos que van más allá de su vida laboral. Si a esto añadimos que la inserción de los varones se produjo de forma preferente en el sector de la construcción, uno de los más castigados por la crisis, frente a la presencia femenina en el empleo de hogar y de cuidados, sector que se ha mantenido más estable aunque precarizado, entenderemos este énfasis en las razones laborales por parte de los varones. Así pues, los relatos recabados reflejan con bastante firmeza las posiciones diferenciadas de ambos géneros en las estructuras sociolaborales de los países de origen y destino, así como las diferencias culturales en torno a su responsabilidad en la familia.

En el caso de Bolivia, la responsabilidad femenina en la atención de la familia está marcada con mucha claridad en los discursos recogidos durante el trabajo de campo. Resulta muy llamativo que las mujeres, al narrar su trayectoria migratoria aluden siempre a la maternidad, a sus responsabilidades en el grupo de parentesco y a los cambios en los ciclos escolares y de vida de sus hijos e hijas. En concreto, 23 de las 25 mujeres entrevistadas han aducido dichas cuestiones mientras que tan sólo 3 de los 13 hombres entrevistados han hecho referencia a la decisión de retornar en relación al ciclo vital de la familia. Además, ninguna de las entrevistadas menciona entre las razones para regresar la creación de un negocio ni tampoco las penurias económicas y laborales que estaban viviendo en España, cuando entre los hombres se encuentran 9 testimonios que construyen la toma de la decisión en términos de desempleo y/o creación de un microemprendimiento en origen. Entre las mujeres bolivianas, los retornos se han producido mayoritariamente porque los hijos no reagrupados se hacen mayores y ya no tienen quien los cuide, porque el padre ha reclamado la tutela legal, porque los hijos estaban descuidados o porque ya no aguantaba más la separación de ellos.

Durante la migración, la organización de la familia debe reconfigurarse y para ello es necesario alcanzar una serie de acuerdos tanto a nivel económico como de cuidados entre la parentela. Cuando esta estructura falla o no se adecua a lo esperado, el retorno aparece como una alternativa, especialmente cuando las condiciones no favorecen la permanencia.

"No, yo no regresé por la crisis económica. Nada, nada... más fue emocional. Se me presentó para la decisión de volverme cuando el padre de mi hija me la estaba quitando y pidiendo a la juez la tutela por abandono. Apenas me enteré de eso no dudé. Directamente dije: "Me retorno". Hice todo lo necesario allá y me vine sobre la marcha” (Cochabamba, Inés, 2010).

En los retornos hacia Ecuador vemos familias que si bien dejaron pequeños en origen, especialmente a cargo de abuelas, finalmente lograron en mayor medida y 
debido al tiempo de permanencia reagruparse en España y abandonar la transferencia transnacional del cuidado ${ }^{4}$. Esto es igualmente común entre las madres separadas o las que se separaron durante la migración, que tras un período de estabilización propiciaron la llegada de sus hijas e hijos. En este sentido, las narrativas muestran que el retorno, además de con la coyuntura de la crisis, se vincula con la fase del ciclo vital en la que se hallan estas familias. El fin de la escolaridad, la independencia económica o residencial respecto a los progenitores, la edad avanzada de los padres o las separaciones y enamoramientos pueden ser claves en el rumbo del proceso migratorio. Lo que está en juego en algunos casos es continuar o separarse de los hijos. La continuidad intergeneracional de la familia es un argumento de peso que desencadena toda una serie de estrategias, entre las cuales figura regresar antes de que los hijos inicien estudios superiores. Este es el caso de Sania, una madre ecuatoriana que llegó a España con su hijo de ocho años y que decide regresar tras permanecer casi una década en España.

“(...) ya se decía que iba a haber una crisis, y qué vamos a hacer los emigrantes, pues que ya no iba ir bien el negocio, todas esas cosas nos, nos hizo pensar en volvernos, aunque mi hijo no, no quería, traer a mi hijo, y también quería que mi hijo siga la universidad aquí, porque yo decía, si me quedo allá pues ya me tocará quedarme unos 5 años de una vez a que él estudie, no. O bien me venía a que el comience aquí, o bien me quedaba allá, definitivamente allá, yo tenía que quedarme para no interrumpir los estudios del chico. $\mathrm{Y}$ al fin me decidí por venir pero me costó mucho, mucho, mucho. Mucho, este me ha costado venirme y me ha costado adaptarme, es decir que el emigrante no es ni de allá, ni de aquí” (Quito, Sania, 2013).

Los relatos de los jóvenes dan, así mismo, cuenta de las fracturas identitarias y relacionales que se producen a su regreso y que sólo tras un período de tiempo se superan. Muchos de estos chicos y chicas hablan con añoranza de España, afirman sentirse bien en el país e imaginan volver a estudiar durante un tiempo en algún momento de su vida.

Así como las migrantes bolivianas enfatizan el papel del cuidado en la decisión de retornar, para las ecuatorianas, cuyos hijos están finalizando o han finalizado ya la etapa escolar, la educación aparece como un elemento clave a la hora de definir el proyecto vital y proyectar el ascenso social a través de la profesionalización de los hijos. Además de la estancia más prolongada de este grupo respecto al boliviano y a los procesos de reunificación de los hijos, esto puede también explicarse por el perfil socioeconómico más elevado de las personas ecuatorianas entrevistadas. En los testimonios de las retornadas ecuatorianas que encabezan un negocio como estrategia de supervivencia, aparece claramente la idea de que el negocio no es una apuesta laboral proyectada sobre los hijos. Se trata más bien de una forma de mantenerse mientras los hijos e hijas finalizan los estudios y llegan a desempeñarse como

${ }^{4}$ Evidentemente este no es siempre el caso. Para un análisis detallado de los esquemas transnacionales de cuidado véase Herrera (2013). 
profesionales asalariados. Estas mujeres, que han vivido en carne propia los efectos de un mercado altamente etnizado y estratificado por género y origen aspiran a otra cosa para sus hijos e hijas (Pedreño 2005). Esto les hace valorar el retorno como alternativa. Tal y como explica una entrevistada, una madre sola que volvió con su hija, hoy empleada como comercial en una empresa, y que montó un negocio de lacado de uñas, "en Europa, lastimosamente, (mi hija) no habría pasado de ser una camarera” (Quito, Wendy, 2012).

El hecho de que los retornos de la población boliviana hayan resultado más inesperados y precarios, en el sentido económico y jurídico, no ha permitido visualizar esa estrategia de proyectar en los hijos la movilidad social de la familia en el retorno. Además, en la mayoría de los casos, al no haber completado su proceso de reagrupación familiar y al interrumpirse su trayectoria migratoria a partir de la crisis, los desgastes afectivos con la familia pueden ser más fuertes que en el caso ecuatoriano y el cumplimiento de los objetivos mucho menor. No obstante, es común la idea de que si se vuelve a migrar se hará en solitario sin implicar a los vástagos. Sin embargo, esta decisión depende del éxito conseguido en España. Para aquellas personas que la migración no mejoró su situación social y económica de partida, la idea de irse de nuevo aparece entre sus pensamientos pero como un proyecto individual que no implica a otros parientes. A ello se une la idea de que lo importante para los hijos es la educación y que su ciclo, al menos secundario, debe de completarse en Bolivia para no cortar la trayectoria escolar. Otra cosa es cuando los hijos se encuentran en edad de estudios universitarios. En esa situación, sobre todo para las personas bolivianas y ecuatorianas que consideran su migración como exitosa, el desplazamiento a España se contempla como una opción muy favorable para mejorar su especialización profesional.

[E- ¿ ¿Tienes pensado regresar a España?] "No, quizás más adelante. Ya cuando mi hijo entre en la Universidad o mi hija, que eso va a ser un gasto más grande para mí. Que su papá me pasa sólo una pensión, no es el sueldo completo" (Cochabamba, Alicia, 2010).

La situación de los hombres solos que estuvieron durante largos periodos separados de familia y que ante la falta de empleo decidieron que era "tiempo de volver” resulta significativa. También en este caso, su proyecto de retorno aparece más asociado a la coyuntura laboral, si bien aducen la lejanía respecto a la familia como un aspecto que tuvieron en cuenta. El regreso de estos varones no ha sido fácil, y como se señala en la literatura (Rosas 2011), ha estado plagado de ambivalencias, tanto en lo que se refiere a su inserción en el trabajo como a su papel de autoridad en la familia y a sus vínculos afectivos con esposas e hijos.

\subsection{IDENTIDADES Y RELACIONES DE GÉNERO EN EL RETORNO CON EMPRENDIMIENTO}

Muchos de los retornados, hombres y mujeres, deciden emprender un negocio. Se trata habitualmente de pequeños comercios o talleres en los que se ve involucrado el conjunto de la familia. Esta modalidad de inversión de los ahorros es, junto a la 
vivienda, un aspecto demostrativo del éxito del proyecto migratorio especialmente atractivo para los varones. Responde al modelo de desarrollo que ha animado una parte de los programas estrella de política pública dirigidos a los migrantes en algunos países latinoamericanos. Entre las iniciativas figura el Fondo Cucayo, provisto por el Estado ecuatoriano entre 2008-2010 y 2010-2012 (Moncayo 2011). Aunque a nivel estatal los programas de retorno en Bolivia son todavía incipientes y con una escasa orientación a promocionar el retorno productivo, este tipo de programas se ha intentado desarrollar a partir de la labor de ONG's. Tal es el caso de ACOBE (Asociación de Cooperación Bolivia-España), que cuenta con programas de apoyo al retorno voluntario productivo, ofreciendo formación, asesoramiento y seguimiento en España y en Bolivia, a través de su contraparte AMIBE (Asociación de Migrantes Bolivia-España) (Parella, Petroff y Serradell 2014). De esta manera, la inversión "productiva" de las remesas, pero también de la fuerza de trabajo de quienes regresan, bien sea a través del aparato gubernamental o asociativo, se concibe como un objetivo prioritario que difumina las condiciones y responsabilidades de los actores institucionales dirigiendo toda la atención sobre los migrantes (García 2004, Cortés 2010, Vega 2014).

En estas iniciativas se proyectan juicios de valor que ponen de relieve el destino preferente respecto al capital acumulado. Sin embargo, la grandilocuencia evocada por el término "emprendimiento" y la altura mítica del "emprendedor" en ocasiones no deja ver con claridad lo modesto de los negocios de estos migrantes. Siguiendo a Newland y Tanaka (2010), el trabajo de campo realizado en ambos contextos permite establecer una diferencia entre quienes emprenden "por oportunidad" y quienes lo hacen "por necesidad". Estos últimos abren pequeños establecimientos o talleres para autoemplearse que descansan en las fuerzas de la familia y proporcionan recursos limitados. Los negocios responden a oficios que expresan las demarcaciones de género: lacado de uñas, peluquerías, almuerzos e internet para ellas, mecánica, cerrajería y soldadura, construcción y transporte para ellos. Con frecuencia los hombres se ocupan del oficio mientas las mujeres están en la trastienda, encargadas del papeleo, los trámites o la comunicación con los clientes. A pesar de esta división en el trabajo, los negocios suelen estar a nombre de los hombres. En general, los más sustentables son los de los varones que detentaban un negocio con anterioridad a la migración; se trata normalmente de hombres que contaban con un oficio, e incluso con redes y clientela ya formadas en entornos locales de vecindario. "Abrí nuevamente mi tienda de barrio que tengo en mi zona -comenta Rosario, una retornada a La Paz-. Tú allá trabajas y ganas y ves tu dinerito. Aquí trabajas para el día, para comer” (La Paz, Rosario, 2012).

Así, la mayoría de quienes emprenden, tanto en Ecuador como en Bolivia, experimenta fuertes límites; algunos han tenido que cerrar por problemas de viabilidad, robos u otras dificultades sobrevenidas. Los responsables de la SENAMI, instancia que regulaba las ayudas en Ecuador, señalan que los solicitantes no siempre tienen experiencia con los negocios y desconocen el terreno y la actividad en la que se aventuran. Su iniciativa responde en muchos casos a la popularización de los beneficios comúnmente asociados a esta forma de autoempleo.

La implicación en el emprendimiento como motivo de retorno también reviste rasgos diferenciales según el género. Por ejemplo en el caso de Bolivia, ninguna de las 
mujeres entrevistadas ha vinculado su regreso a un sentimiento empresarial, que sin embargo, sí es una razón que destacan los entrevistados durante el proceso de la toma de la decisión. Además, cuando aparece el emprendimiento femenino, tras el retorno y no necesariamente para el retorno, éste suele relacionarse con las necesidades familiares y no con proyecciones empresariales propias. En algunos casos, estas inversiones son omitidas debido a que la creación del negocio no se produjo para ellas mismas sino que ofrecieron sus ahorros para tal fin a los padres de sus hijos o a sus propios vástagos. Alicia, una de nuestras entrevistadas, comenta: "Y ahora cuando vine, mi hijo me dijo: 'Mamá, ya sé manejar'. Así que compramos un auto. Y ahora mi hijo ya trabaja con el auto. Compramos una línea de taxi” (Cochabamba, Alicia, 2010).

En las entrevistas en Ecuador destacan algunas mujeres, casos emblemáticos para el Fondo Cucayo, de auténticas emprendedoras por oportunidad con perspectivas de proyección en el negocio, pero aún en estos casos, las mujeres no conciben la iniciativa como una mera fuente de ingresos, sino que entretejen de un modo virtuoso negocio y familia. El negocio permite articular los distintos capitales humanos que la familia alberga con el fin de reunirla tras un periodo de dispersión. Marianita, costurera antes de migrar, ha planificado y encabeza el regreso escalonado de los que partieron, y su vinculación con los miembros que se quedaron. Para ello ha optimizado empleos, inversiones, deudas, jubilaciones y fuerza de trabajo con vistas a reunir a todas sus hijas y yernos en torno a un taller textil con alguna empleada.

"Si ya acabamos de pagar ese préstamo de mi hijo, él ya se siente más libre, ya puede endeudarse en otra cosa, porque lo que queremos es sacarnos una bordadora. Si mi hijo regresa, entonces mi hijo se encargaría de la bordadora, porque él tiene que estudiar, porque ya está viendo en Madrid los diseños de bordadoras para él aprender, porque si él viene acá, ya se endeudaría en la bordadora y él se pondría al frente de eso, porque tampoco va a venir acá a la desocupación, porque no estamos acostumbrados. Él no está para que diga, que mi mamita me mantenga, porque aquí saben coser todos mis hijos, hombres y mujeres, porque yo toda la vida he cosido" (Quito, Marianita, 2012).

No obstante, y dejando al margen estos casos, habitualmente el emprendimiento es una estrategia de autoempleo de alcance limitado que permite la supervivencia del hogar. Para ellos puede ser también un lugar de sostén y autoestima, mientras que para ellas es o bien una estrategia para sostener la formación de sus hijas e hijos como profesionales o una forma de articular a la familia en destino, especialmente en aquellos casos en que los miembros quedaron separados por la migración. Algunas mujeres bolivianas entienden el negocio como una compensación a los padres de sus hijos por haberse hecho cargo de los mismos durante su ausencia. Es el caso de Cristina, quien regresó a su casa porque el padre de su hijo la amenazaba constantemente con quitarle la custodia ya que el niño quedó a cargo de una hermana materna. Aunque el padre no participaba económica ni emocionalmente en los cuidados del más pequeño, reclamaba su paternidad y pretendía denunciar a Cristina por el abandono de su hogar durante la migración. A pesar de este nivel de tensiones en la pareja ella menciona cómo ayudó económicamente dos veces a su marido para poder tener la tranquilidad de estar trabajando en el extranjero sin problemas. Sin duda, 
estamos ante una estrategia para negociar la movilidad internacional.

"Yo le mandé a mi esposo casi tres meses de trabajo. Le mandé casi 3.000 euros para que pague una línea de bus como le llaman en España. Yo me compré un lote (un terreno) para construir una casita pero no me alcanzó el dinero para construir. Porque de ahí mi marido se operó y le he tenido que dar 2.500 euros” (Cochabamba, Cristina, 2010).

Esta situación evidencia que la migración, si bien supone un cuestionamiento de las funciones que las mujeres desempeñan dentro de la familia, también visualiza que muchas de ellas intentan compensar la ruptura de esos patrones hegemónicos. Las transferencias económicas de Cristina hacia el padre de su hijo pueden considerarse como una manera de apaciguar el desafío que en términos de roles de género supuso su migración. Dicha inversión puede ser observada, tal y como señala Herrera (2012a), como una forma de explotación económica encubierta por la ideología de género y de parentesco pero en algunos casos también puede considerarse una manera de revertir la imagen negativa que se ha construido en Bolivia sobre las mujeres migrantes especialmente aquellas que son madres. Estigmatizadas y culpabilizadas por dejar a sus hijos en origen (Pedone, 2008), estas mujeres pueden utilizar estas inversiones para demostrar que las mejoras laborales y económicas del grupo doméstico dependen de su provisión material. Por eso Cristina recuerda a su pareja su capacidad como sustentadora: "Ahora yo le digo: 'Si tú quieres hacer tus cosas (se refiere a cuando le es infiel) pues agarra tu ropa y vete. Vete que yo aquí a estar feliz o si no llamo a mi jefe y regreso a España"” (Cochabamba, Cristina, 2010). La participación laboral de las mujeres bolivianas representa en alguna medida cambios en sus relaciones de pareja introduciendo en algunos casos una renegociación de las relaciones de género. Sin embargo, tal y como otras investigaciones han puesto en evidencia anteriormente (Bastia 2011b), estas alteraciones en los roles pocas veces derivan en auténticas reconfiguraciones de la organización familiar.

El trabajo de campo en Ecuador revela que cuando los hombres regresan tras períodos prolongados y se sitúan al frente de estos negocios se produce una devaluación de los ingresos en el hogar. Esto, unido a la renegociación de papeles y autoridades en la pareja y con los hijos es una fuente potencial de conflictos.

"Solo, solo ese fue el grande error, haberme ido solo (...) el esfuerzo que se ha hecho no, no, mi esposa en este caso no entiende, o sea parece, dice que me he ido a disfrutar pero no es así, es doloroso comenzar desde cero, o sea es muy difícil, entonces es, eso es así no hay nada más que agregar” (Quito, Eduardo, 2013).

$\mathrm{Al}$ igual que se ha detectado en otros estudios, también en éste, los varones se quejan de la incomprensión de sus compañeras tras largos años de envío de remesas, mientras que las mujeres no ven reconocido su aporte en destino al tiempo que resienten la devaluación de la situación económica al inicio del nuevo negocio o cuando éste no prospera como esperaban (Rosas 2011, Parella 2012). 


\section{CONCLUSIONES}

El análisis de las migraciones de retorno de las personas ecuatorianas y bolivianas residentes en España en el contexto de crisis económica pone de manifiesto la influencia de los factores laborales y familiares en la toma de la decisión y el modo en que éstos difieren en relación al género y al grado de asentamiento de ambos colectivos en la sociedad de destino. Para ambos sexos el retorno va más allá del empleo y los bienes materiales adquiridos y se vincula al ciclo de vida que experimenta la familia y sus miembros. Sin embargo, en la medida en que hombres y mujeres mantienen posiciones, obligaciones y responsabilidades desiguales dentro del grupo de parentesco, es de esperar que sus motivaciones para regresar a casa sean también distintas y que estén marcadas por una ideología de género patriarcal.

De esta manera, mayoritariamente las mujeres vinculan su vuelta con aspectos relacionados con la salud y los cuidados en sus familias de origen. Sobre todo en el caso de las mujeres bolivianas se hace pertinente su regreso debido a fallos y tensiones surgidos en la organización de los cuidados. Las razones de las mujeres ecuatorianas no son ajenas a este discurrir afectivo pero también incluyen elementos de oportunidades educativas y profesionales dirigidas a sus hijos. Estos últimos se muestran inicialmente reacios a volver a un país que apenas conocen y en el que finalmente acaban por acomodarse. La diferencia de énfasis entre ambos colectivos femeninos debe vincularse al distinto grado de asentamiento de ambas nacionalidades en España y las consiguientes ventajas económicas que éste implica. La proyección de Ecuador como un país de oportunidades puede estar desempeñando un papel a la hora de animar, que no de decidir, el camino de retorno. La población ecuatoriana, mucho más instalada en destino, con una media de diez años de residencia y, en muchos casos, con la nacionalidad en la mano o con un permiso de larga duración ya había completado su proceso de reagrupación familiar por lo que en el caso de las familias con hijos, éstos ya se encontraban reunidos en destino. En estos casos, el retorno ha implicado desmontar la vida de la unidad familiar y sus miembros, hecho al que se han resistido, al menos en la primera fase de la crisis. Los flujos de llegada de la población boliviana fueron más tardíos en el tiempo (2005-2007) y todavía se estaban estableciendo en el momento en el que se impone el visado y estalla la crisis. Tal es así que la mayor parte de las personas entrevistadas carecían de permisos de residencia y prácticamente no se habían planteado la reunificación de la familia en España.

Por otra parte, los hombres asocian más su decisión de regresar a motivos económicos y profesionales aunque la dimensión familiar también está detrás de sus motivaciones, ya que su situación laboral determina su rol de proveedores económicos, pero, sobre todo, su status en la comunidad. En este sentido, es habitual que las narrativas masculinas hagan más referencia que la de las mujeres a las vicisitudes de la crisis económica y la precariedad laboral vivida en España a partir de la etapa de destrucción de empleo masivo. No solamente porque ésta afectase especialmente a las actividades laborales en las que ellos se insertaron, sino también por la influencia que 
la pérdida de empleo o la merma en las ganancias esperadas tienen sobre su posición de autoridad dentro del grupo de parentesco. Por este motivo, son los hombres los que evocan en mayor medida, la creación de un negocio como un motivo de regreso al hogar y como estrategia de reinserción tras la vuelta, si bien advertimos que se trata de fórmulas de autoempleo de subsistencia y necesidad que de inversiones por oportunidad. Así, su proyección renovada como proveedores a través del retorno, en no pocos casos se encuentra con fracasos o con un horizonte limitado, hecho que influencia su masculinidad.

\section{REFERENCIAS BIBLIOGRÁFICAS}

ANDERSON, BRIDGET (2000): Doing the Dirty Work: The Global Politics of Domestic Labour. London: Zed Books.

ANTHIAS, FLOYA y GABRIELLA LAZARIDIS (2000): Gender and migration in Southern Europe: women on the move. New York: Berg

ARIAS, PATRICIA (2013): Migración, economía campesina y ciclo de desarrollo doméstico. Discusiones y estudios recientes. Estudios Demográficos y Urbanos. Vol.28 (1), 93-121.

BABY-COLLIN, VIRGINA y GENEVIEVE CORTÉS (2014): New trends in Bolivian migratory fields in the face of the crisis. Revista CIDOB d'Afers Internacionals, 106-107: 61-83.

BASTIA, TANJA (2014): Intersectionality, migration and development. Progress in Development Studies 14(3): 237-248, DOI: 10.1177/1464993414521330

- (2011a): Should I stay or should I go? Return migration in times of crises. Journal of International Development, 23: 583-595, DOI: 10.1002/jid.1794

- (2011b): Migrations as protest? Negotiating gender, class and ethnicity in urban Bolivia. Environment and Planning A, 43: 1514-1529, DOI: 10.1068/a43365

BERTOLI, SIMONE y JESÚS FERNÁNDEZ-HUERTAS (2013): Multilateral resistence to migration. Journal of Development Economics, 102: 79-100, DOI: 10.1016/j.jdeveco.2012.12.001

GAMBURD, MICHELE RUTH (2000): The Kitchen Spoon's Handle: Transnationalism and Sri Lanka's Migrant Housemaids. Cornell University Press.

CASSARINO, JEAN PIERRE (2004): Theorising return migration: the conceptual approach to return migrants revisited. International Journal on Multicultural Societies, 6(2): 253-279.

CELLERI, DANIELA (2011): Jóvenes Indígenas (kichwa-otavalos) entre etnicidad, clase y género. Boletín Científico Sapiens Research, 1(2): 13-15. 
COLECTIVO IOÉ y MERCEDES FERNÁNDEZ (2010): Encuesta nacional de inmigrantes 2007: el mercado de trabajo y las redes sociales de los inmigrantes. Madrid: Ministerio del Trabajo e Inmigración.

COLECTIVO IOÉ. (2012): Impactos de la crisis sobre la población inmigrante. Madrid: OIM.

CONSTABLE, NICOLE (1999): At home but not at home: Filipina narratives of ambivalent Returns. Cultural Anthropology, 14(2): 203-228.

CORTÉS, ALMUDENA (2010): La reinvención del nexo migración y desarrollo desde el sur de Europa: el caso de Ecuador y España. Relaciones Internacionales, 14, 3959.

DE HAAS, HEIN (2012): The migration and development pendulum: a critical view on research and policy international migration. Internacional Migration, 50(3): 825, DOI: 10.1111/j.1468-2435.2012.00755.x

DE LA TORRE, LEONARDO (2012): "Más notas sobre el retorno cíclico boliviano”. En: C. Solé et al. (eds.), Migración internacional y espacios sociales transnacionales: Una aproximación a las estrategias migratorias de la población boliviana, Barcelona: Anthropos.

GARCÍA, RODOLFO (2003): Seminario Internacional La Transferencia y Uso de las Remesas: Proyectos Productivos y de Ahorro. México: Zacatecas, CEPAL, Sin Fronteras, UAZ.

GUARNIZO, LUIS (1997): “'Going Home’: Class, Gender and Household Transformation among Dominican Return Migrants”. En: Pessar, P. (ed.) Caribbean Circuits. New Directions in the Study of Caribbean Migration. New York: Center for Migration Studies, 13-60.

HANSEN, PETER (2008): Circumcising migration: gendering return migration among Somalilanders. Journal of Ethnic and Migration Studies, 34(7): 1109-25. (DOI: 10.1080/13691830802230422).

HERNÁNDEZ KATTYA et al. (2012). Entre crisis y crisis: experiencias de emigración y retorno. El caso de los barrios populares de noroccidente de Quito. Quito: Abya-Yala.

HERRERA, GIOCONDA (2012). Starting over again? Crisis, gender and social reproduction among Ecuadorian migrants in Spain. Feminist Economics, 18(2): 125-148.(DOI: 10.1080/13545701.2012.688997)

- (2013): Gender and international migration: contributions and cross-fertilizations. Annual Review of Sociology, 39: 471-489, DOI: 10.1146/annurev-soc-071811145446

HERRERA, GIOCONDA y VEGA, CRISTINA (Coords.) (2013): El retorno a Ecuador desde España: estrategias económicas, socio-familiares y afectivas para una integración transnacional. Quito: FLACSO Ecuador. 
HERRERA, GIOCONDA, MONCAYO, MARÍA ISABEL y ESCOBAR, ALEXANDRA, (2012): Perfil Migratorio del Ecuador 2011. Quito: Organización Internacional para las Migraciones (OIM).

HOFMANN, ERIN TROUTH (2014). Does Gender Ideology Matter in Migration?

International Journal of Sociology, 44(3): 23-41, DOI: 10.2753/IJS00207659440302

IZQUIERDO, MARIA (2013): “La socialización de género. La construcción social del género”. En: Díaz Martínez y Dema Moreno (eds.) Sociología y género, Madrid, Tecnos.

IZQUIERDO, ANTONIO y RAQUEL MARTÍNEZ-BUJÁN (2014): From Favourites to 'With no Return': Permanence and Mobility of Latin American Immigration in Spain during the $21^{\text {st }}$ Century. Canadian Ethnic Studies, 46(3): 103-130.

JONES, RICHARD y LEONARDO DE LA TORRE (2011): Diminished tradition of return? Transnational migration in Bolivia's Valle Alto. Global Networks, 11(2): 180-202, DOI: 10.1111/j.1471-0374.2011.00314.X

LEDO, CARMEN (2014): Múltiples arreglos del hogar transnacional en la ciudad de Cochabamba. Revista CIDOB d'Afers Internationals, 106-107: 105-128.

MAHLER, SARAH y PATRICIA PESSAR (2001): Gendered geographies of power: analyzing gender across transnational spaces. Identities, 7(4): 441-459, DOI: 10.1080/1070289X.2001.9962675

MARTÍNEZ-BUJÁN, RAQUEL (2015): Gendered motivations for migration return to Bolivia from Spain. Journal of Immigrants and Refugee Studies, 13(4): 401-418.

MASSEY, DOUGLAS (1990). Social structure, household strategies and the cumulative causation of migration, Population Index, 56(1): 3-26, DOI: $10.2307 / 3644186$

MONCAYO, MARÍA ISABEL (2011). Políticas de retorno en América Latina: miradas cruzadas. Andina Migrante, 10, 2-10.

MOROKVASIC, MIRJANA (1984). Birds of passage are also women. Women in Migration, 18 (4), 886-907, DOI: 10.2307/2546066

MOSCOSO, MARÍA FERNANDA (2013): Biografía para uso de los pájaros: memoria, infancia y migración. Quito: IAEN.

NEWLAND, KATHLEEN y HIROYUKI TANAKA (2010). Mobilizing diaspora entrepreneurship for development. Washington, D.C: Migration Policy Institute.

OLWIG, KAREN FOG (2012): The 'successful' return: Caribbean narratives of migration, family and gender. Journal of the Royal Anthropology Institute, 18: 828845, DOI: 10.1111/j.1467-9655.2012.01794.X

PARELLA SONIA, ALISA PETROFF Y OLGA SERRADELL (2014): Programas de retorno voluntario en Bolivia y España en contextos de crisis. Revista CIDOB d'afers internacionals, 106-107, 1133-6595. 
PARELLA, SONIA (2012): Familia transnacional y redefinición de los roles de género. El caso de la migración boliviana en España. Papers, 97 (3), 661-684.

PEDONE, CLAUDIA (2008): 'Varones aventureros' vs. 'madres que abandonan': reconstrucción de las relaciones familiares a partir de la migración ecuatoriana. RеMHи 16(30): 45-64.

- (2004): Tú siempre jalas a los tuyos: cadenas y redes migratorias de las familias ecuatorianas hacia España. Tesis doctoral en Universidad Autónoma de Barcelona.

PEDREÑO, ANDRÉS (2005): “La condición inmigrante: exploraciones e investigaciones desde la región de Murcia”. Revista Andaluza de Ciencias Sociales, (5), 114-116.

- (2013): 'Que no sean como nosotros': Trayectorias formativo- laborales de los hijos de familias inmigrantes en el campo murciano. Murcia: EDITUM..

PLEWA, PIOTR (2009): Voluntary Return Programmes: Could they assuage the effects of the economic crisis? Working Paper, 75. Centre of Migration, Policy and Society. University of Oxford.

PORTES, A. et al. (1999): The study of transnacionalism: pitfalls and promises of an emergent research field. Ethnic and Racial Studies, 22(2): 217-37. (DOI: 10.1080/014198799329468)

RIVERA, LILIANA, (2011): "¿Quiénes son los retornados? Apuntes sobre el migrante retornado en el México contemporáneo". En: Bela Feldman-Bianco et al. (comps.) La construcción social del sujeto migrante en América Latina: prácticas, representaciones y categorías. Quito, Ecuador: CLACSO, FLACSO y Universidad Alberto Hurtado

ROSAS, CAROLINA (2011): De nuevo bajo el mismo techo... Desafíos y reconfiguraciones en los procesos de reunificación de parejas migrantes. Mora, 18 (2) $s / n$.

VEGA, CRISTINA (2014): El retorno más allá del mito del emprendedor. Presentado en el seminario 'La migración de retorno en Ecuador: nuevos y viejos desafíos', Quito.

VLASE, IONELA (2013). My husband is a patriot!: Gender and Romanian family return migration from Italy. Journal of Ethnic and Migration Studies, 35(5): 741758, DOI: 10.1080/1369183X.2013.756661. 